\title{
Analytical Solution for Thermal Transport in Packed Beds with
}

3

4 include a spatially and temporally constant volumetric heat source. Our solution enables 208:405-416) is enough to calculate fluid and particle temperatures.

\section{Volumetric Heat Source}

anstitute of Process and Particle Engineering, Graz University of Technology, Inffeldgasse 13/III, 8010 Graz, Austria

bResearch Center Pharmaceutical Engineering GmbH, Inffeldgasse 13/III, 8010 Graz, Austria

*Corresponding author: E-mail: radl@tugraz.at; Tel.: +43 316873 30412; Fax: +43 316 8731030412

\section{Abstract}

We present an analytical solution for the thermal transport in fluid-particles systems that the rapid calculation of temperature profiles in systems undergoing chemical reactions or phase change phenomena. Also, we propose a map that helps in deciding in which situations the simple solution of Schumann (Journal of the Franklin Institute 1929, 


\section{Introduction}

2 The thermal design of process equipment in various industrial applications, such as solar

3 power plants (Behar et al., 2013), thermal energy storage (Van Lew et al., 2011), and

4 reactive systems ( $\mathrm{Li}$ et al., 2016) is an essential engineering task. Often, heat exchange

5 between the fluid (often a gas) and the particles in the presence of a heat source has to be

6 considered. This makes the solution of the set of equations challenging, often calling for a

7 numerical solution. An analytical solution to predict the behaviour of such systems can be

8 useful when (i) verifying the correctness of such numerical solutions, as well as (ii) when

9 developing advanced control strategies that require an extremely fast evaluation of model

10 equations (Rehrl et al., 2016). A number of attempts to derive analytical solutions were

11 successful for certain simplified situations: Schumann (1929) presented such a solution

12 for transient heat transfer in a one-dimensional packed bed. Even though his solution is

13 valid only for perfectly insulated systems without heat source, it has been extensively

14 used by various researchers (Anderson et al., 2015; Cascetta et al., 2014; Li et al., 2014;

15 Valmiki et al., 2012; Van Lew et al., 2011; Xu et al., 2012; Xu et al., 2015). White and

16 Korpela (1979) obtained an exact solution for the temperature distribution in a perfectly

17 insulated packed bed (for various initial and boundary conditions) using a Laplace

18 transformation and the method of characteristics. Murata $(1971,1983)$ calculated the

19 temperature distribution based on Schumann's method considering heat conduction

20 within the solid particles. Similarly, Villatoro et al. (2009) provided an approximate

21 analytical solution for such systems.

22 Another route was followed in the work of Amundson (1956); he attempt to predict the

23 temperature distribution in moving and fixed beds including a volumetric heat source.

24 Although a heat source was already considered in his work, he assumed that the heat 
1 Later, Sundaresan et al. (1980) somewhat refined this work, however still failing to derive

2 a solution for systems involving a heat source. Later, Sözen and Vafai (1990) claimed that

3 the derivation of analytical solutions for predicting temperatures in the packed beds with

4 evaporation (i.e., a negative heat source) is impossible.

5 We will demonstrate in our present contribution that the above statement of Sözen and

6 Vafai (1990) is not accurate in case of a spatially and temporally fixed evaporation rate.

7 Thus, we present an analytical solution that is indeed useful for a wide number of systems

8 in which the volumetric heat source is constant. In detail, we extend the analytical solution

9 presented by Schumann (1929) to consider a constant heat source in the solid phase. This

10 will be realized via solving the set of heat transfer equations for the gas and solid phase

11 in the packed bed using Laplace transformation.

Another key effort in the recent past was to develop numerical strategies to tackle heat transfer problems in chemical reaction engineering applications. Among the plethora of 14 numerical strategies that have been developed, the so-called "Computational Fluid Dynamics-Discrete Element Method" (CFD-DEM) is one of the most attractive strategies. This method allows (i) studies of packed or fluidized beds, as well as (ii) a direct modelling 17 of the particle phase (Askarishahi et al.; Deen and Kuipers, 2014; Lattanzi and Hrenya, 2016; Li et al., 2016; Patil et al., 2015; Sutkar et al., 2016). However, a simulation based on the CFD-DEM still requires comparison of methods relying on a continuum versus a discrete representation of the particles to avoid unwanted artefacts caused by the discrete representation of the system. Thus, considering the intrinsic limitations of a numerical solution, it appears that an analytical solution for heat exchange in the fluidparticle system with a heat source would be helpful. 


\section{Theoretical Development}

2.1. Packed Bed Heat Transfer Model

3 We considered the transient heat up of gas and particles (with diameter $d_{p}$ ) in a packed

4 bed with a fixed voidage $\varepsilon g$ subject to a fixed volumetric heating rate $\dot{q}$. All physical

5 properties (i.e., the density $\rho$ and the heat capacity $C_{p}$ ) of the gas and particles are assumed

6 to be constant and independent of the temperature. After neglecting gas dispersion and

7 heat conduction in the particle bed, the following differential heat balance equations for

8 the gas and the particle phase are considered:

$$
\begin{aligned}
& \varepsilon_{g} \rho_{g} C_{p, g} \frac{\partial T_{g}^{\prime}}{\partial t}=-\varepsilon_{g} \rho_{g} C_{p, g} u_{g} \frac{\partial T_{g}^{\prime}}{\partial z}-h a\left(T_{g}^{\prime}-T_{p}^{\prime}\right) \\
& \left(1-\varepsilon_{g}\right) \rho_{p} C_{p, p} \frac{\partial T_{p}^{\prime}}{\partial t}=h a\left(T_{g}^{\prime}-T_{p}^{\prime}\right)+\dot{q}
\end{aligned}
$$

9 The above equations state that the rate of enthalpy change per unit total volume ffor each

10 phase) equals the volumetric heat transfer rate, and the heat inflow due to convection. In

11 the above equations, the specific surface area $a$ in $\left[\mathrm{m}^{2} / \mathrm{m}_{\mathrm{tot}}{ }^{3}\right]$ is defined as:

$$
a=\left(1-\varepsilon_{g}\right) 6 / d_{p}
$$

$12 h$ is the heat transfer coefficient in, which is allowed to depend on the flow conditions, but

13 is assumed to be constant throughout the bed. The dimensionless temperatures are

14 defined as

$$
\begin{aligned}
& T_{g}=\frac{T_{g}^{\prime}-T_{g, 0}^{\prime}}{T_{g, i}^{\prime}-T_{g, 0}^{\prime}} \\
& T_{p}=\frac{T_{p}^{\prime}-T_{g, 0}^{\prime}}{T^{\prime}{ }_{g, i}-T^{\prime}{ }_{g, 0}}
\end{aligned}
$$

15 The inlet boundary and initial conditions are respectively defined as:

$$
T_{g}(0, t)=1
$$




$$
\begin{aligned}
& T_{g}(x, 0)=0 \\
& T_{p}(x, 0)=0
\end{aligned}
$$

1 For simplification, we next define

$$
\begin{aligned}
& h_{g}=\frac{h a}{\varepsilon_{g} \rho_{g} C_{p, g}}=\frac{6 h\left(1-\varepsilon_{g}\right)}{\varepsilon_{g} \rho_{g} C_{p, g} d_{p}} \\
& h_{p}=\frac{h a}{\left(1-\varepsilon_{g}\right) \rho_{p} C_{p, p}}=\frac{6 h}{\rho_{p} C_{p, p} d_{p}} \\
& h_{q}=\frac{\dot{q}}{\left(1-\varepsilon_{g}\right) \rho_{p} C_{p, p} \Delta T^{\prime}}
\end{aligned}
$$

2 We then re-write the transport equations to arrive at

$$
\begin{aligned}
& \frac{\partial T_{g}}{\partial t}=-u_{g} \frac{\partial T_{g}}{\partial z}-h_{g}\left(T_{g}-T_{p}\right) \\
& \frac{\partial T_{p}}{\partial t}=h_{p}\left(T_{g}-T_{p}\right)+h_{q}
\end{aligned}
$$

3 Note that the constants $h_{g}, h_{p}$ and $h_{q}$ have the units [1/s]. It is now natural to identify

4 dimensionless time and space coordinates as $t^{*}=h_{p}\left(t-z / u_{g}\right)$ and $z^{*}=h_{g} z / u_{g}$, 5 respectively. Thus, $u_{g} / h_{g}$ is a characteristic thermal length. Also, Eqns. 12 and 13 reveal 6 the key dimensionless influence parameters which are (i) a volumetric heat capacity 7 ratio $h^{*}=\frac{h_{g}}{h_{p}}=\frac{\left(1-\varepsilon_{g}\right) \rho_{p} C_{p, p}}{\varepsilon_{g} \rho_{g} C_{p, g}}$, and (ii) a dimensionless heating rate $h_{q}^{*}=\frac{h_{q}}{h_{p}}$. In what follows, 8 however, we will first avoid introducing these dimensionless quantities, and analyse the 9 problem given by Eqns. 12 and 13 in its original form. We will return to the dimensionless representation when presenting our results in Chapter 3. In order to solve the above set of partial differential equations, Laplace transformation can be used. The Laplace transform of the unknown function $T_{g}(t, z)$ (and similarly for $T_{p}$ ), as well as its time derivative, are given by 


$$
\begin{aligned}
& \ell\left\{T_{g}(t, z)\right\}=\bar{T}_{g}(s, z)=\int_{0}^{\infty} e^{-s t} T_{g}(t, z) d t \\
& \ell\left\{\frac{\partial T_{g}(t, z)}{\partial t}\right\}=s \bar{T}_{g}(s, z)-T_{g}(0, z)
\end{aligned}
$$

1 Hence, Laplace transformation of Eqns. 12 and 13 leads to:

$$
\begin{aligned}
& s \overline{T_{g}}-T_{g}(0, z)=-u_{g} \frac{\partial \overline{T_{g}}}{\partial z}-h_{g}\left(\overline{T_{g}}-\overline{T_{p}}\right) \\
& s \overline{T_{p}}-T_{p}(0, z)=h_{p}\left(\overline{T_{g}}-\overline{T_{p}}\right)+\frac{h_{q}}{s}
\end{aligned}
$$

2 Since $T_{g}(0, z)$ and $T_{p}(0, z)$ are zero, Equation 17 can be simplified as:

$$
\overline{T_{p}}=\frac{h_{p} \overline{T_{g}}+\frac{h_{q}}{s}}{s+h_{p}}
$$

3 By substituting Equation 18 in equation 16, we arrive at

$$
\frac{s}{u_{g}}\left(1+\frac{h_{g}}{s+h_{p}}\right) \overline{T_{g}}+\frac{\partial \overline{T_{g}}}{\partial z}=\frac{h_{g}}{\left(s+h_{p}\right) u_{g}} \frac{h_{q}}{s}
$$

4 For simplification we now define

$$
\alpha=\frac{s}{u_{g}}\left(1+\frac{h_{g}}{s+h_{p}}\right)
$$

5 Using the integration factor $e^{\alpha z}$, Equation 19 is rewritten as

$$
\frac{\partial}{\partial z}\left[e^{\alpha z} \bar{T}_{g}\right]=e^{\alpha z} \frac{h_{g}}{\left(s+h_{p}\right) u_{g}} \frac{h_{q}}{s}
$$

6 The solution of this ODE is straight forward and, after considering the boundary condition

$7 \quad T_{g, i}$ for $T_{g}$ at $z=0$, leads to

$$
\overline{T_{g}}=\frac{T_{g, i}}{s} e^{-\alpha z}-\frac{h_{q} h_{g}}{s\left(s+h_{p}\right) u_{g}} \frac{1}{\alpha} e^{-\alpha z}+\frac{h_{q} h_{g}}{s\left(s+h_{p}\right) u_{g}} \frac{1}{\alpha}
$$


1 After some substitutions and replacements, the introduction of a scaled bed position $t_{c}=$

$2 z / u_{g}$, and an inverse Laplace transformation, we arrive at:

$$
\begin{aligned}
& T_{g}\left(t, t_{c}\right)= \\
& T_{g, i} e^{-t_{c} h_{g}} e^{-h_{p}\left(t-t_{c}\right)} J_{0}\left(2 \sqrt{-a_{0}\left(t-t_{c}\right)}\right) \\
& +a_{q}\left[\left(h_{g}+h_{p}\right) t-1+e^{-\left(h_{g}+h_{p}\right) t}\right] \\
& +e^{-t_{c} h_{g}}\left[T_{g, i} h_{p}-a_{q}^{\prime}\left(h_{p}\left(t-t_{c}\right)+1\right)+a_{q} h_{p}\right] I_{1} \\
& +e^{-t_{c} h_{g}}\left[a_{q}^{\prime} h_{p} I_{2}+a_{q} h_{g} e^{-\left(h_{p}+h_{g}\right)\left(t-t_{c}\right)} I_{3}\right] \\
& T_{p}\left(t, t_{c}\right)= \\
& -a_{q}^{\prime \prime}\left[\frac{1-e^{-h_{p} t}}{h_{p}}-\left(h_{p}+h_{g}\right)\left(-\frac{1}{h_{p}{ }^{2}}+\frac{1}{h_{p}} t+\frac{1}{h_{p}{ }^{2}} e^{-h_{p} t}\right)\right. \\
& \left.-\frac{\left\{e^{-h_{p} t}-e^{-\left(h_{p}+h_{g}\right) t}\right\}}{h_{g}}\right]+\frac{h_{q}}{h_{p}}\left(1-e^{-h_{p} t}\right) \\
& +e^{-t_{c} h_{g}}\left[T_{g, i} h_{p}-a^{\prime \prime}{ }_{q}\left(\left(h_{p}+h_{g}\right)\left(t-t_{c}\right)-1\right)\right] I_{1} \\
& +e^{-t_{c} h_{g}}\left[a^{\prime \prime}{ }_{q}\left(h_{p}+h_{g}\right) I_{2}-a^{\prime \prime}{ }_{q} e^{-\left(h_{p}+h_{g}\right)\left(t-t_{c}\right)} I_{3}\right]
\end{aligned}
$$

3 Where we have used the following definitions:

$$
\begin{aligned}
& a_{0}=t_{c} h_{g} h_{p} ; a_{q}=\frac{h_{g} h_{q}}{\left(h_{g}+h_{p}\right)^{2}} ; a_{q}^{\prime}=\frac{h_{g} h_{q}}{h_{g}+h_{p}} ; a^{\prime \prime}{ }_{q}=\frac{h_{p} h_{g} h_{q}}{\left(h_{p}+h_{g}\right)^{2}} \\
& I_{1}=\int_{0}^{t-t_{c}} e^{-h_{p} u} J_{0}\left(2 \sqrt{-a_{0} u}\right) d u ; I_{2}=\int_{0}^{t-t_{c}} u e^{-h_{p} u} J_{0}\left(2 \sqrt{-a_{0} u}\right) d u ; \\
& I_{3}=\int_{0}^{t-t_{c}} e^{h_{g} u} J_{0}\left(2 \sqrt{-a_{0} u}\right) d u
\end{aligned}
$$

4 Details related to the above derivation are comprehensively presented in Appendix A. We

5 note in passing that a simple quadrature method was applied to approximate the integrals

6 in equation 25. 


\subsection{Benchmarking the Analytical Solution}

3 After successful calculation of the gas and particle temperatures in the packed bed, their

4 time profiles were studied for different heating rates. First, the heating rate was set to zero to investigate pure heat exchange between the gas and the particles. This situation is

6 identical to that considered by Schumann (1929), and a comparison with the Schumann

7 result proved the correct limiting behaviour of our solution. Second, a scenario involving

8 a negative heat source (e.g., due to an endothermic reaction, or evaporation of a liquid

9 from the particle surface) was considered. For this situation the set of governing equations was discretized and solved using MATLAB® (specifically, the function "pdepe" was used). The temperatures obtained from this numerical calculation and our analytical solution were then compared. This study revealed that the deviation between these two solutions for the particle temperatures was larger than that for the gas temperatures. Hence, only results for particle temperature are depicted in Figure 1, which shows the time evolution of the error for this temperature. In this figure we have also included a line

16 for a relative accuracy of $0.1 \%$ (i.e., $E_{T_{P}}=\left|\frac{T_{P}^{\text {Numerical }}-T_{P}^{\text {analytical }}}{T_{P}^{\text {analytical }}}\right|=10^{-3}$ ) which acts as the acceptance criterion for the comparison of the numerical and analytical solution. As expected, grid refinement decreases the deviation from the analytical solution at the cost of computation time: in case we use between 10 and 200 discretization points it takes between $10 s$ and $650 s$ to evaluate the discretized set of governing equations in MATLAB $^{\circledR}$. In contrast, the evaluation of Eqns. 23 and 24 takes less than 5 s in any situation studied here. Clearly, and as can be seen in Figure 1, only the solution using more 200 grid cells yields acceptable results (i.e., for which the relative deviation is less than $10^{-4}$ ). Thus, our analytical solution is by at least a factor of 100 faster than a numerical approach, even though we have to numerically approximate the integrals in Eqns. 23 and 24. 
After successful calculation of the temperatures, CFD-DEM code was developed to take a

4 heat source located on the particles' surface into account. Specifically, we implemented all

5 relevant models into the tool $\operatorname{CFDEM}^{\circledR}$ (Goniva et al., 2012). Several simulations were performed using the developed $\mathrm{CFDEM}^{\circledR}$ tool considering various heating rates, and the

7 results were compared with that obtained from our analytical solution (see Figure 2).

8 Clearly, gas and particle temperatures are in good agreement in most regions of the bed,

9 and systematic deviations are primarily observed near the inlet region. After a careful analysis of these deviations (see Appendix C for details), we conclude that they are due to the Lagrangian-to-Eulerian mapping of the particle volumes: this mapping leads to tiny fluctuation of the local voidage in the particle bed, which are then amplified by the strong dependency of the local heat transfer coefficient on the voidage. Also, it can be observed from Figure 2 that the deviations for the gas temperature are more pronounced compared to that for the particle temperature. Moreover, an increase in the rate of heat exchange causes a larger deviation between our analytical solution and predictions by CFD-DEM simulations. This is expected, since the main error introduced in a CFD-DEM simulation is due to the above discussed mapping, and hence the predicted local heat transfer coefficient.

21 We next consider a map spanned by all relevant dimensionless system parameters that indicates solutions close to that of Schumann, i.e., situations with very weak heat sources. Such a map is especially helpful when deciding whether the heat release rate should be considered in a model or not. 
3 Particle and gas temperatures were calculated using the analytical solution for a large

4 array of combinations of the dimensionless heating rate $h_{q}^{*}=\frac{h_{q}}{h_{p}}$ and the volumetric heat

5 capacity ratio $h^{*}=\frac{h_{g}}{h_{p}}=\frac{\varepsilon_{p} \rho_{p} C_{p, p}}{\varepsilon_{g} \rho_{g} C_{p, g}}$. The calculation was performed for a variety of

6 dimensionless times $t^{*}$ in a packed bed with total length $z^{*}=h_{g} z_{b e d} / u_{g}$. We have then

7 determined the critical dimensionless heating rate which leads to a maximum relative

8 error of $20 \%$ of the predicted gas or particle temperature when using the Schumann

9 solution. This maximum error occurs at the outlet of the bed, i.e., at $\mathrm{z}=\mathrm{zbed}$, and hence it

10 is essential to consider the bed length in what follows.

11 As depicted in Figure 3 (panel a), for a fixed volumetric heat capacity ratio and bed length,

12 the critical dimensionless heating rate is smaller for the particle temperature compared

13 to the gas temperature. Thus, the error in the particle temperature limits the applicability

14 of Schumann's solution when applying his result to a system with volumetric heat source.

15 Therefore, in what follows we only consider the particle temperature, and results of these

16 calculations are depicted in Figure 3 (panel b). It can be easily seen from this figure that 17 at identical dimensionless heating rates the volumetric heat capacities ratio only mildly

18 affects the limiting curve of $20 \%$ deviation from the Schumann solution. Specifically, an

19 increase in the heat capacity ratio leads to only a weak increase in the critical

20 dimensionless heating rate. This is due to the fact that higher volumetric heat capacity

21 ratios reflect a system in which particles have a higher capacity to store the heat. Thus,

22 the thermal inertia of the system is simply greater.

23 Another point discerned from Figure 3 (panel b) is that the critical dimensionless heating 24 rate saturates with time, and that all curves for different $h^{*}$ collapse for long times. Thus, 
1 as we approach the steady-state solution, only the dimensionless heating rate (for a fixed

2 bed length) determines the particle temperature in the bed. In other words, and after

3 recalling the definition of $h_{q}^{*}=\frac{\dot{q} d_{p}}{6 h\left(1-\varepsilon_{g}\right) \Delta T}$, this means that the competition of heat

4 release and heat transfer determines the particle temperature for long times. This is also

5 expected from a simple steady-state analysis considering the particles only. Interestingly,

6 the critical heating rate is always smaller for early times, i.e., before the steady state

7 solution is reached. This simply means that relative errors during the heat up phase of the

8 bed are larger than that when seeking a steady-state solution.

9 As can be anticipated from our arguments in the last paragraph, the range of $\mathrm{h}_{\mathrm{q}}^{*}$ in which

10 Schumann solution is valid is strongly affected by the dimensionless height of the bed. In

11 Figure 3 (panel c) the corresponding map for $h_{q}^{*}$ is depicted for $h^{*}=10$. We note that for

12 larger values of $\mathrm{h}^{*}$ the results change only marginally, such that the data shown in Figure

$133 \mathrm{c}$ represents the limit of infinitely high heat capacity ratios. As shown in this figure, at

14 higher dimensionless height, $\mathrm{z}^{*}$, the domain of validity for both $\mathrm{t}^{*}$ and $\mathrm{h}_{\mathrm{q}}^{*}$ is narrower.

15 Again, this can be easily explained by the increase of the bed temperature along the flow

16 direction at steady-state conditions.

Figure 3

\section{Conclusion}

The set of heat transfer equation was analytically solved, utilizing Laplace transformation, for a one-dimensional packed bed with constant volumetric heat source. The correctness of the calculated temperature profiles was proved via a comparison with numerical predictions based on $\mathrm{MATLAB}^{\circledR}$. The calculated temperature profiles were then also compared to CFD-DEM-based simulations. This study illustrated the need to improve CFD-DEM mapping schemes, since already minute errors in the predicted local 
1 voidfraction result in considerable errors when estimating the heat transfer coefficient, and hence the local temperatures.

Since Schumann's solution (i.e., a solution that does not consider a heat source) is extensively used in the literature, we also made an attempt to generate a map of situation in which Schumann's solution is acceptable for heated systems. It was demonstrated that at a constant dimensionless heating rate, an increase in the thermal capacity ratio $h^{*}$ makes Schumann's solution valid for a wider range of non-dimensional times. However, this effect levels off at $h^{*}=10$, and vanishes for long times. Most important, the bed length critically affects the applicability of Schumann's solution.

\section{Acknowledgement}

SR acknowledges partial funding for the present research from EC's FP7 Grant agreement no. 604656 (NanoSim). CFDEM ${ }^{\circledR}$ is a registered trade mark of DCS Computing GmbH, the producer of the $\mathrm{CFDEM}^{\circledR}$ coupling software. MA acknowledges Boehringer Ingelheim pharmaceutical company for funding this research project.

\section{References}

Amundson, N.R., 1956. Solid-fluid interactions in fixed and moving beds fixed beds with small particles. Industrial \& Engineering Chemistry 48, 26-35. Anderson, R., Bates, L., Johnson, E., Morris, J.F., 2015. Packed bed thermal energy storage: Askarishahi, M., Salehi, M.-S., Radl, S., Full-Physics Simulations of Spray-Particle Interaction in a Bubbling Fluidized Bed. Submitted to AIChE Journal. Behar, O., Khellaf, A., Mohammedi, K., 2013. A review of studies on central receiver solar thermal power plants. Renewable and Sustainable Energy Reviews 23, 12-39.

Cascetta, M., Cau, G., Puddu, P., Serra, F., 2014. Numerical investigation of a packed bed thermal energy storage system with different heat transfer fluids. Energy Procedia 45, 598-607.

Deen, N., Kuipers, J., 2014. Direct Numerical Simulation (DNS) of mass, momentum and heat transfer in dense fluid-particle systems. Current Opinion in Chemical Engineering 5, 84-89.

Goniva, C., Kloss, C., Deen, N.G., Kuipers, J.A., Pirker, S., 2012. Influence of rolling friction on single spout fluidized bed simulation. Particuology 10, 582-591. Lattanzi, A.M., Hrenya, C.M., 2016. A coupled, multiphase heat flux boundary condition for the discrete element method. Chemical Engineering Journal 304, 766-773. 
Li, P., Xu, B., Han, J., Yang, Y., 2014. Verification of a model of thermal storage incorporated with an extended lumped capacitance method for various solid-fluid structural combinations. Solar Energy 105, 71-81.

Li, Z., van Sint Annaland, M., Kuipers, J., Deen, N., 2016. Effect of superficial gas velocity on the particle temperature distribution in a fluidized bed with heat production. Chemical Engineering Science 140, 279-290.

Murata, S., 1971. EXTENSION OF SCHUMANN"S THEORY TO THE CASE OF LOW THERMAL DIFFUSIVITY OF SOLID PARTICLES. Journal of Chemical Engineering of Japan 4, 140-146.

Murata, S., 1983. Exact Analytical Solutions to the Extension of Schumann's Theory on the Heat Transfer in Packed Bed. Journal of the Faculty of Agriculture, Kyushu University 27, 151-158.

Patil, A., Peters, E., Kuipers, J., 2015. Comparison of CFD-DEM heat transfer simulations with infrared/visual measurements. Chemical Engineering Journal 277, 388-401.

Rehrl, J., Kruisz, J., Sacher, S., Khinast, J., Horn, M., 2016. Optimized continuous pharmaceutical manufacturing via model-predictive control. International Journal of Pharmaceutics 510, 100-115.

Schumann, T.E., 1929. Heat transfer: a liquid flowing through a porous prism. Journal of the Franklin Institute 208, 405-416.

Sözen, M., Vafai, K., 1990. Analysis of the non-thermal equilibrium condensing flow of a gas through a packed bed. International Journal of Heat and Mass Transfer 33, 1247-1261. Sundaresan, S., Amundson, N.R., Aris, R., 1980. Observations on fixed-bed dispersion models: The role of the interstitial fluid. AIChE Journal 26, 529-536.

Sutkar, V.S., Deen, N.G., Patil, A.V., Salikov, V., Antonyuk, S., Heinrich, S., Kuipers, J., 2016. CFD-DEM model for coupled heat and mass transfer in a spout fluidized bed with liquid injection. Chemical Engineering Journal 288, 185-197.

Valmiki, M., Karaki, W., Li, P., Van Lew, J., Chan, C., Stephens, J., 2012. Experimental investigation of thermal storage processes in a thermocline tank. Journal of solar energy engineering 134, 041003.

Van Lew, J.T., Li, P., Chan, C.L., Karaki, W., Stephens, J., 2011. Analysis of heat storage and delivery of a thermocline tank having solid filler material. Journal of solar energy engineering 133, 021003.

Villatoro, F., Pérez, J., Domínguez-Muñoz, F., Cejudo-López, J., 2009. Approximate analytical solution for the heat transfer in packed beds for solar thermal storage in building simulators, Eleventh International IBPSA Conference,(July 2009), pp. 709-715. White, H.C., Korpela, S.A., 1979. On the calculation of the temperature distribution in a packed bed for solar energy applications. Solar Energy 23, 141-144.

$\mathrm{Xu}, \mathrm{B} ., \mathrm{Li}, \mathrm{P.}-\mathrm{W} ., \mathrm{Chan}, \mathrm{C} . \mathrm{L} ., 2$ 2012. Extending the validity of lumped capacitance method for large Biot number in thermal storage application. Solar Energy 86, 1709-1724.

$\mathrm{Xu}, \mathrm{B}$., Li, P., Chan, C., 2015. Application of phase change materials for thermal energy storage in concentrated solar thermal power plants: a review to recent developments. Applied Energy 160, 286-307. 


\section{Appendix A - Inverse Laplace Transformation}

2 A1. Gas Temperature

3 The inverse Laplace transform of the first term in right hand side of equation 22, denoted

4 as $\bar{T}_{g 1}$, can be calculated considering that

$$
\bar{T}_{g 1}=e^{-t_{c} s\left(1+\frac{h_{g}}{s+h_{p}}\right)} \frac{T_{g, i}}{S}=\frac{T_{g, i}}{S} e^{-t_{c} s} e^{-t_{c} h_{g}} e^{t_{c} h_{g}\left(\frac{h_{p}}{s+h_{p}}\right)}
$$

5 Note, that we used the scaled bed position $t_{c}=z / u_{g}$ in the above equation as described

6 in the manuscript.

$7 \quad$ By defining $a_{0}=t_{c} h_{g} h_{p}, T_{g 1}$ is given by

$$
T_{g 1}=T_{g, i} e^{-t_{c} h_{g}} \ell^{-1}\left\{e^{-t_{c} s}\left[\frac{1}{s} e^{\left(\frac{a_{0}}{s+h_{p}}\right)}\right]\right\}
$$

8 Considering

$$
\begin{aligned}
& \ell^{-1}\left\{e^{-a s} \bar{F}(s)\right\}= \begin{cases}F(t-a) & t>a \\
0 & t<a\end{cases} \\
& \ell^{-1}\{\bar{F}(s-a)\}=e^{a t} F(t)
\end{aligned}
$$

9

10 This equation can be rewritten as

$$
T_{g 1}=T_{g, i} e^{-t_{c} h_{g}} F_{1}\left(t-t_{c}\right)
$$

12 Where the function $\mathrm{F}$ is represented by 


$$
\begin{aligned}
& F_{1}(t)=\ell^{-1}\left\{\left[\frac{1}{s} e^{\left(\frac{a_{0}}{s+h_{p}}\right)}\right]\right\}=\ell^{-1}\left\{\left[\frac{s+h_{p}}{s} \frac{1}{s+h_{p}} e^{\left(\frac{a_{0}}{s+h_{p}}\right)}\right]\right\} \\
& \quad=\int_{0}^{t} H_{1}(t-u) G_{1}(u) d u \\
& G_{1}(u)=\ell^{-1}\left\{\left[\frac{1}{s+h_{p}} e^{\left(\frac{a_{0}}{s+h_{p}}\right)}\right]\right\}=e^{-h_{p}} \ell^{-1}\left\{\frac{1}{s} e^{\frac{a_{0}}{s}}\right\}=e^{-h_{p} u} J_{0}\left(2 \sqrt{-a_{0} u}\right) \\
& H_{1}(u)=\ell^{-1}\left\{\left[\frac{s+h_{p}}{s}\right]\right\}=\ell^{-1}\left\{\left[1+\frac{h_{p}}{s}\right]\right\}=\delta(u)+h_{p} \\
& F_{1}\left(t-t_{c}\right)=\int_{0}^{t-t_{c}}\left[\delta\left(t-t_{c}-u\right)+h_{p}\right] e^{-h_{p} u} J_{0}\left(2 \sqrt{-a_{0} u}\right) d u
\end{aligned}
$$

2 Here $J_{0}$ denotes the Bessel function of order zero. Therefore, the inverse Laplace

3 transform for first term in right hand side is calculated as 4

$$
T_{g 1}=T_{g, i} e^{-t_{c} h_{g}} \int_{0}^{t-t_{c}}\left[\delta\left(t-t_{c}-u\right)+h_{p}\right] e^{-h_{p} u} J_{0}\left(2 \sqrt{-a_{0} u}\right) d u
$$

5 For the calculation of the second term in right hand side of Equation 22 we first rewrite 6 this expression as the sum of three sub-terms:

$$
\begin{aligned}
\bar{T}_{g 2}=-e^{-t_{c} s\left(1+\frac{h_{g}}{s+h_{p}}\right)}\left[h_{q} h_{g} \frac{1}{s^{2}} \frac{1}{s+h_{g}+h_{p}}\right] \\
=-e^{-t_{c} s} e^{-t_{c} h_{g}} e^{\left(\frac{a_{0}}{s+h_{p}}\right)} \frac{h_{q} h_{g}}{\left(h_{g}+h_{p}\right)^{2}}\left[\frac{h_{g}+h_{p}}{s^{2}}-\frac{1}{s}+\frac{1}{s+h_{g}+h_{p}}\right] \\
=\bar{T}_{g 21}+\bar{T}_{g 22}+\bar{T}_{g 23}
\end{aligned}
$$


2 By defining $a_{q}=\frac{h_{q} h_{g}}{\left(h_{g}+h_{p}\right)^{2}}$, and $a_{q}^{\prime}=\frac{h_{q} h_{g}}{h_{g}+h_{p}}$, the inverse Laplace transform of $\bar{T}_{g 21}$ is given 3 by

$$
\begin{aligned}
T_{g 21}=-a_{q}^{\prime} \ell^{-1}\left\{\frac{1}{s^{2}} e^{-t_{c} s} e^{-t_{c} h_{g}} e^{\left(\frac{a_{0}}{s+h_{p}}\right)}\right\} \\
=-a_{q}^{\prime} e^{-t_{c} h_{g}} \ell^{-1}\left\{e^{-t_{c} s}\left[\frac{1}{s^{2}} e^{\left(\frac{a_{0}}{s+h_{p}}\right)}\right]\right\}=-a_{q}^{\prime} e^{-t_{c} h_{g}} F_{21}\left(t-t_{c}\right)
\end{aligned}
$$

4 Where the function $F_{21}$ is

$$
\begin{gathered}
F_{21}(t)=\ell^{-1}\left\{\left[\frac{1}{s^{2}} e^{\left(\frac{a_{0}}{s+h_{p}}\right)}\right]\right\}=\ell^{-1}\left\{\left[\frac{s+h_{p}}{s^{2}} \frac{1}{s+h_{p}} e^{\left(\frac{a_{0}}{s+h_{p}}\right)}\right]\right\} \\
=\int_{0}^{t} H_{21}(t-u) G_{21}(u) d u
\end{gathered}
$$

5

6 and the functions $G_{21}(u)$ and $H_{21}(u)$ are given by

$$
\begin{aligned}
& G_{21}(u)=\ell^{-1}\left\{\left[\frac{1}{s+h_{p}} e^{\left(\frac{a_{0}}{s+h_{p}}\right)}\right]\right\}=e^{-h_{p} u} \ell^{-1}\left\{\frac{1}{s} e^{\frac{a_{0}}{s}}\right\}=e^{-h_{p} u} J_{0}\left(2 \sqrt{-a_{0} u}\right) \\
& H_{21}(u)=\ell^{-1}\left\{\left[\frac{s+h_{p}}{s^{2}}\right]\right\}=\ell^{-1}\left\{\left[\frac{1}{s}+\frac{h_{p}}{s^{2}}\right]\right\}=1+h_{p} u
\end{aligned}
$$

7

8 Therefore, $T_{g 21}$ is given by

$$
T_{g 21}=-a_{q}^{\prime} e^{-t_{c} h_{g}} \int_{0}^{t-t_{c}}\left[h_{p}\left(t-t_{c}-u\right)+1\right] e^{-h_{p} u} J_{0}\left(2 \sqrt{-a_{0} u}\right) d u
$$


1 The inverse Laplace transform of $\bar{T}_{g 22}$ is, similar to $\bar{T}_{g 1}$, calculated by

$$
\begin{aligned}
T_{g 22}=a_{q} \ell^{-1} & \left\{\frac{1}{s} e^{-t_{c} s} e^{-t_{c} h_{g}} e^{\left(\frac{a_{0}}{s+h_{p}}\right)}\right\}=a_{q} e^{-t_{c} h_{g}} \ell^{-1}\left\{e^{-t_{c} s}\left[\frac{1}{s} e^{\left(\frac{a_{0}}{s+h_{p}}\right)}\right]\right\} \\
& =a_{q} e^{-t_{c} h_{g}} F_{1}\left(t-t_{c}\right)
\end{aligned}
$$

2 Thus, the inverse Laplace transform of $\bar{T}_{g 22}$ is given by

$$
T_{g 22}=a_{q} e^{-t_{c} h_{g}} \int_{0}^{t-t_{c}}\left[\delta\left(t-t_{c}-u\right)+h_{p}\right] e^{-h_{p} u} J_{0}\left(2 \sqrt{-a_{0} u}\right) d u
$$

3 Considering the last term in Equation A11, we can write

$$
\begin{aligned}
T_{g 23}=-a_{q} \ell^{-1} & \left\{\frac{1}{s+h_{g}+h_{p}} e^{-t_{c} s} e^{-t_{c} h_{g}} e^{\left(\frac{a_{0}}{s+h_{p}}\right)}\right\} \\
= & -a_{q} e^{-t_{c} h_{g}} e^{-1}\left\{e^{-t_{c} s}\left[\frac{s+h_{p}}{\left(s+h_{g}+h_{p}\right)} \frac{1}{\left(s+h_{p}\right)} e^{\left(\frac{a_{0}}{s+h_{p}}\right)}\right]\right\} \\
= & -a_{q} e^{-t_{c} h_{g}} F_{23}\left(t-t_{c}\right)
\end{aligned}
$$

4 Where $F_{23}(t)$ is

$$
F_{23}(t)=\int_{0}^{t} H_{23}(t-u) G_{23}(u) d u
$$

5

$$
\begin{aligned}
& G_{23}(u)=\ell^{-1}\left\{\frac{1}{s+h_{p}} e^{\left(\frac{a_{0}}{s+h_{p}}\right)}\right\}=e^{-h_{p} t} \ell^{-1}\left\{\frac{1}{s} e^{\frac{a_{0}}{s}}\right\}=e^{-h_{p} u} J_{0}\left(2 \sqrt{-a_{0} u}\right) \\
& H_{23}(u)=\ell^{-1}\left\{\frac{s+h_{p}}{s+h_{g}+h_{p}}\right\}=\ell^{-1}\left\{1-\frac{h_{g}}{s+h_{g}+h_{p}}\right\}=\delta(u)-h_{g} e^{-\left(h_{p}+h_{g}\right) u}
\end{aligned}
$$




$$
\begin{aligned}
T_{g 23}=-a_{q} e^{-t_{c} h_{g}} \int_{0}^{t-t_{c}}\left[\delta\left(t-t_{c}-u\right)\right. \\
\left.\quad-h_{g} e^{-\left(h_{p}+h_{g}\right)\left(t-t_{c}-u\right)}\right] e^{-h_{p} u} J_{0}\left(2 \sqrt{-a_{0} u}\right) d u
\end{aligned}
$$

1 Finally, the inverse Laplace transform of the third term in Eqn. 22, denoted here as $\bar{T}_{g 3}$, is

2 calculated as

$$
T_{g 3}=a_{q} \ell^{-1}\left\{\frac{h_{g}+h_{p}}{s^{2}}-\frac{1}{s}+\frac{1}{s+h_{g}+h_{p}}\right\}=a_{q}\left[\left(h_{g}+h_{p}\right) t-1+e^{-\left(h_{g}+h_{p}\right) t}\right]
$$

3

4 After summation of all terms, $T_{g}\left(t, t_{c}\right)$ is written as:

$$
\begin{array}{lr}
T_{g}\left(t, t_{c}\right)=T_{g 1}+\left(T_{g 21}+T_{g 22}+T_{g 23}\right)+T_{g 23}= & \text { (A25) } \\
T_{g, i} e^{-t_{c} h_{g}} \int_{0}^{t-t_{c}}\left[\delta\left(t-t_{c}-u\right)+h_{p}\right] e^{-h_{p} u} J_{0}\left(2 \sqrt{-a_{0} u}\right) d u & \left\{T_{g 1}\right\} \\
-a_{q}^{\prime} e^{-t_{c} h_{g}} \int_{0}^{t-t_{c}}\left[h_{p}\left(t-t_{c}-u\right)+1\right] e^{-h_{p} u} J_{0}\left(2 \sqrt{-a_{0} u}\right) d u & \left\{T_{g 21}\right\} \\
+a_{q} e^{-t_{c} h_{g}} \int_{0}^{t-t_{c}}\left[\delta\left(t-t_{c}-u\right)+h_{p}\right] e^{-h_{p} u} J_{0}\left(2 \sqrt{-a_{0} u}\right) d u & \left\{T_{g 22}\right\} \\
-a_{q} e^{-t_{c} h_{g}} \int_{0}^{t-t_{c}}\left[\delta\left(t-t_{c}-u\right)-h_{g} e^{-\left(h_{p}+h_{g}\right)\left(t-t_{c}-u\right)}\right] e^{-h_{p} u} J_{0}\left(2 \sqrt{-a_{0} u}\right) d u & \left\{T_{g 23}\right\} \\
+a_{q}\left[\left(h_{g}+h_{p}\right) t-1+e^{-\left(h_{g}+h_{p}\right) t}\right] & \left\{T_{g 3}\right\}
\end{array}
$$

5 After rearranging Eqn. A25, as well as considering that the integrals involving a product

6 with the delta function yield the function value itself, $T_{g}\left(t, t_{c}\right)$ is calculated as (see also

$7 \quad$ Eqn. 23 in the manuscript):

$$
\begin{aligned}
& T_{g}\left(t, t_{c}\right)= \\
& T_{g, i} e^{-t_{c} h_{g}} e^{-h_{p}\left(t-t_{c}\right)} J_{0}\left(2 \sqrt{-a_{0}\left(t-t_{c}\right)}\right)
\end{aligned}
$$




$$
\begin{aligned}
& +a_{q}\left[\left(h_{g}+h_{p}\right) t-1+e^{-\left(h_{g}+h_{p}\right) t}\right] \\
& +e^{-t_{c} h_{g}}\left[T_{g, i} h_{p}-a_{q}^{\prime}\left(h_{p}\left(t-t_{c}\right)+1\right)+a_{q} h_{p}\right] I_{1} \\
& \left.+e^{-t_{c} h_{g}\left[a^{\prime}{ }_{q} h_{p} I_{2}+a_{q} h_{g} e^{-\left(h_{p}+h_{g}\right)\left(t-t_{c}\right)}\right.} I_{3}\right]
\end{aligned}
$$

1 Where we have used the following definitions:

$$
\begin{aligned}
& I_{1}=\int_{0}^{t-t_{c}} e^{-h_{p} u} J_{0}\left(2 \sqrt{-a_{0} u}\right) d u ; I_{2}=\int_{0}^{t-t_{c}} u e^{-h_{p} u} J_{0}\left(2 \sqrt{-a_{0} u}\right) d u ; \\
& I_{3}=\int_{0}^{t-t_{c}} e^{h_{g} u} J_{0}\left(2 \sqrt{-a_{0} u}\right) d u
\end{aligned}
$$

3 A2. Particle Temperature Calculation

4 We now recall the Laplace transform of the particle temperature given by Eqn. 18, and the 5 solution for the gas temperature available in Eqn. 22:

$$
\begin{gathered}
\overline{T_{p}}=\frac{T_{g, i}}{s\left(s+h_{p}\right)} e^{-t_{c} s\left(1+\frac{h_{g}}{s+h_{p}}\right)}-h_{q} h_{g} h_{p} \frac{1}{s^{2}\left(s+h_{p}\right)\left(s+h_{g}+h_{p}\right)} e^{-t_{c} s\left(1+\frac{h_{g}}{s+h_{p}}\right)} \\
+h_{q} h_{g} h_{p} \frac{1}{s^{2}\left(s+h_{p}\right)\left(s+h_{g}+h_{p}\right)}+\frac{h_{q}}{s\left(s+h_{p}\right)}
\end{gathered}
$$

6

7 To obtain the inverse Laplace transform of $\overline{T_{p}}$, we split the above expression into four 8 terms

$$
\overline{T_{p}}=\bar{T}_{p_{1}}+\bar{T}_{p_{2}}+\bar{T}_{p_{3}}+\bar{T}_{p_{4}}
$$

9

10 By rewriting the first term in right hand side of Equation $A 28, \bar{T}_{p_{1}}$ is given by 


$$
\bar{T}_{p_{1}}=T_{g, i} e^{-t_{c} s} e^{-t_{c} h_{g}} e^{t_{c} h_{g}\left(\frac{h_{p}}{s+h_{p}}\right)}\left[\frac{1}{s}-\frac{1}{\left(s+h_{p}\right)}\right]
$$

1 Considering the time shifting property of a Laplace transformation, the above equation

2 can be rewritten as

$$
\bar{T}_{p_{1}}=T_{g, i} e^{-t_{c} h_{g}} F_{1}\left(t-t_{c}\right)
$$

3 Where

$$
F_{1}(t)=\ell^{-1}\left\{e^{\left(\frac{a_{0}}{s+h_{p}}\right)}\left[\frac{1}{s}-\frac{1}{\left(s+h_{p}\right)}\right]\right\}
$$

4 By implementing the convolution theorem on the above equation, we obtain

$$
F_{1}(t)=\int_{0}^{t} H_{1}(t-u) G_{1}(u) d u-e^{-h_{p} t} J_{0}\left(2 \sqrt{-a_{0} t}\right)
$$

5 where

$$
\begin{aligned}
& G_{1}(u)=\ell^{-1}\left\{\left[\frac{1}{s+h_{p}} e^{\left(\frac{a_{0}}{s+h_{p}}\right)}\right]\right\}=e^{-h_{p}} \ell^{-1}\left\{\frac{1}{s} e^{\frac{a_{0}}{s}}\right\}=e^{-h_{p} u} J_{0}\left(2 \sqrt{-a_{0} u}\right) \\
& H_{1}(u)=\ell^{-1}\left\{\left[\frac{s+h_{p}}{s}\right]\right\}=\ell^{-1}\left\{\left[1+\frac{h_{p}}{s}\right]\right\}=\delta(u)+h_{p}
\end{aligned}
$$

6

7 By substituting $H_{1}$ and $G_{1}$ in Equation A31, $T_{p_{1}}$ can be calculated as

$$
\begin{gathered}
T_{p_{1}}(t)=T_{g, i} e^{-t_{c} h_{g}}\left[\int_{0}^{t-t_{c}}\left(\delta\left(t-t_{c}-u\right)+h_{p}\right) e^{-h_{p} u} J_{0}\left(2 \sqrt{-a_{0} u}\right) d u\right. \\
\left.-e^{-h_{p}\left(t-t_{c}\right)} J_{0}\left(2 \sqrt{-a_{0}\left(t-t_{c}\right)}\right)\right]
\end{gathered}
$$


1 Using the same methodology and partial fractional decomposition, $\bar{T}_{p_{2}}$ is given by

$$
\bar{T}_{p_{2}}=-a_{q}^{\prime \prime} e^{-t_{c} s} e^{-t_{c} h_{g}} e^{\left(\frac{a_{0}}{s+h_{p}}\right)} \frac{1}{s+h_{p}}\left[\frac{h_{p}+h_{g}}{s^{2}}-\frac{1}{s}+\frac{1}{s+h_{p}+h_{g}}\right]
$$

2 Where $a_{q}^{\prime \prime}=\frac{h_{p} h_{g} h_{q}}{\left(h_{p}+h_{g}\right)^{2}}$. Hence, we arrive at:

$$
\begin{array}{r}
T_{p_{2}}(t)=-a^{\prime \prime}{ }_{q} e^{-t_{c} h_{g}} \int_{0}^{t-t_{c}}\left[\left(h_{p}+h_{g}\right)\left(t-t_{c}-u\right)-1\right. \\
\left.+e^{-\left(h_{p}+h_{g}\right)\left(t-t_{c}-u\right)}\right] e^{-h_{p} u} J_{0}\left(2 \sqrt{-a_{0} u}\right) d u
\end{array}
$$

3

4 Finally the inverse Laplace transform of $\bar{T}_{p_{3}}$ and $\bar{T}_{p_{4}}$ is given by

$$
\begin{aligned}
& T_{p_{3}}(t)=-a^{\prime \prime}{ }_{q} {\left[\frac{1-e^{-h_{p} t}}{h_{p}}-\left(h_{p}+h_{g}\right)\left\{-\frac{1}{h_{p}{ }^{2}}+\frac{1}{h_{p}} t+\frac{1}{h_{p}{ }^{2}} e^{-h_{p} t}\right\}\right.} \\
&-\left.\frac{\left\{e^{-h_{p} t}-e^{-\left(h_{p}+h_{g}\right) t}\right\}}{h_{g}}\right] \\
& T_{p_{4}}(t)=\frac{h_{q}}{h_{p}}\left(1-e^{-h_{p} t}\right)
\end{aligned}
$$

5 After summation of all terms, the particle temperature is calculated as

$$
T_{p}\left(t, t_{c}\right)=T_{p 1}+T_{p 2}+T_{p 3}+T_{p 4}
$$

6

$$
\begin{aligned}
& T_{p}\left(t, t_{c}\right) \\
& =
\end{aligned}
$$




$$
\begin{gathered}
T_{g, i} e^{-t_{c} h_{g}}\left[\int_{0}^{t-t_{c}}\left(\delta\left(t-t_{c}-u\right)+h_{p}\right) e^{-h_{p} u} J_{0}\left(2 \sqrt{-a_{0} u}\right) d u\right. \\
\left.-e^{-h_{p}\left(t-t_{c}\right)} J_{0}\left(2 \sqrt{-a_{0}\left(t-t_{c}\right)}\right)\right] \\
-a^{\prime \prime}{ }_{q} e^{-t_{c} h_{g}} \int_{0}^{t-t_{c}}\left[\left(h_{p}+h_{g}\right)\left(t-t_{c}-u\right)-1\right. \\
\left.+e^{-\left(h_{p}+h_{g}\right)\left(t-t_{c}-u\right)}\right] e^{-h_{p} u} J_{0}\left(2 \sqrt{-a_{0} u}\right) d u \\
-a^{\prime \prime}{ }_{q}\left[\frac{1-e^{-h_{p} t}}{h_{p}}-\left(h_{p}+h_{g}\right)\left\{-\frac{1}{h_{p}{ }^{2}}+\frac{1}{h_{p}} t+\frac{1}{h_{p}{ }^{2}} e^{-h_{p} t}\right\}\right. \\
\left.-\frac{\left\{e^{-h_{p} t}-e^{-\left(h_{p}+h_{g}\right) t}\right\}}{h_{g}}\right] \\
+\frac{h_{q}}{h_{p}}\left(1-e^{-h_{p} t}\right)
\end{gathered}
$$

2 After rearranging Eqn. A41, as well as simplifying the integrals involving the delta

3 function as explained above, $T_{p}\left(t, t_{c}\right)$ is calculated as (see also Eqn. 24 in the manuscript):

$$
\begin{aligned}
& T_{p}\left(t, t_{c}\right)= \\
& -a^{\prime \prime}{ }_{q}\left[\frac{1-e^{-h_{p} t}}{h_{p}}-\left(h_{p}+h_{g}\right)\left(-\frac{1}{h_{p}^{2}}+\frac{1}{h_{p}} t+\frac{1}{h_{p}{ }^{2}} e^{-h_{p} t}\right)\right. \\
& \left.-\frac{\left\{e^{-h_{p} t}-e^{-\left(h_{p}+h_{g}\right) t}\right\}}{h_{g}}\right]+\frac{h_{q}}{h_{p}}\left(1-e^{-h_{p} t}\right) \\
& +e^{-t_{c} h_{g}}\left[T_{g, i} h_{p}-a_{q}^{\prime \prime}\left(\left(h_{p}+h_{g}\right)\left(t-t_{c}\right)-1\right)\right] I_{1} \\
& +e^{-t_{c} h_{g}}\left[a^{\prime \prime}{ }_{q}\left(h_{p}+h_{g}\right) I_{2}-a^{\prime \prime}{ }_{q} e^{-\left(h_{p}+h_{g}\right)\left(t-t_{c}\right)} I_{3}\right]
\end{aligned}
$$

5 Note that we have used the definitions in Eqn. A27 for the integral terms $I_{1}, I_{2}$, and $I_{3}$. 


\section{Appendix B - Octave Scripts for Evaluation of the Solution}

3 In order to make the presented analytical solution easy to use, the computer code (using

4 Matlab®-compatible scripts for the open-source tool "Octave",

5 https://www.gnu.org/software/octave) for evaluation of the temperature profiles is

6 provided. To calculate the temperature, one needs to run the script

7 "plotScript_packedBed.m" in Octave (all functions detailed below must be added to the

8 path using the "addpath" command). Note that all input parameters required to calculate

9 the temperature have been described via comments in this script. The result plots will be saved in a file entitled "temperature_xxx_hEvap_yyy.png" in which "xxx" represents the

11 methodology to calculate the profile (i.e. Laplace transformation or the Schumann solution), and "yyy" represents the value of the evaporation rate.

The computer code consists of the following computer code:

1. The main script entitled "plotScript_packedBed.m", which is used to input all input parameters, call all relevant functions, and plot the results.

2. A set of functions that perform the calculations:

a. function "NusseltDeenEtAl.m" for calculation of the heat transfer coefficient using the correlation developed by Deen et al. (2012).

b. function "packedBedTemperatureLT.m" for calculation of temperatures using the presented method in the current study, i.e., a Laplace transformation.

c. function "packedBedIntegral.m" to calculate the integral terms using a numerical approximation.

d. function "packedBedTrnsTemperatures.m" to calculate the temperatures using the classical method presented by Schumann. This function uses the sub-functions "packedBedTrnsM0.m", "packedBedTrnsMn.m" and "packedBedTrnsMnSum.m". 
1 Deen, N.G., Kriebitzsch, S.H., van der Hoef, M.A., Kuipers, J., 2012. Direct numerical 2 simulation of flow and heat transfer in dense fluid-particle systems. Chemical 3 Engineering Science 81, 329-344.

Appendix C - CFD-DEM Simulation Details and Benchmark against the

\section{Schumann Solution}

The simulation setup, as well as physical properties and simulation condition for the studied system are summarized in Table C.1. A 3D CFD-DEM simulation was performed using CFDEM® code (Kloss et al., 2012). The cell size in each direction was considered as $2 d_{P}$. It should be mentioned that in case of enforced simulation, the width, length, and height of the bed was $6 \times 6 \times 28 d_{P}$ (i.e., somewhat larger than in Table C.1), and the cell size in each direction was set to $1 d_{P}$. Also, particles were placed on a hexahedral lattice into the simulation box such that every particle was perfectly centered in each cell. This was done to enforce a uniform particle volume fraction in each cell, i.e., $\varphi_{P}=\pi / 6$.

Table C.1 - Simulation and physical properties for the CFD-DEM simulations.

In order to address the deviation of the temperature predicted when using a CFD-DEM simulation from the analytical solution, two additional simulation scenarios were considered: i) a simulation using the above described lattice initialization of the particles in the bed, which enforces a perfectly uniform distribution of voidage in the bed; as well as ii) a voidage calculation based on a simplified mapping method (for randomly arranged particles), in which the volume fraction of solid particles in each cell was calculated based on the particle whose centres reside inside that cell. The latter differs from the standard method used in the CFDEM ${ }^{\circledR}$ code, which uses a more advanced "divided" mapping method.

The predicted temporal evolution of the gas and particle temperature for both scenarios is depicted in Figure $\mathrm{C} 1$ showing data at two different positions in the bed. We note in passing that the dimensionless bed position in this figure is slightly different due to the differences in 
1 the bed voidage (causing different fluid speed and heat transfer coefficient). It can be easily

2 discerned that when using the lattice distribution (see panel a in Figure C1), the predicted 3 temperature is in excellent agreement with the corresponding analytical value. This

4 demonstrates that the heat exchange between gas and particle, as well as heat source/sink for 5 particles have been accurately implemented in the $\mathrm{CFDEM}^{\circledR}$ code. The small deviation 6 observed in Figure C1a can be attributed to (i) numerical diffusion inherent when using a finite 7 computational grid, and (ii) the fluctuation of the velocity experienced by the first row of 8 particles. The latter causes a small error in the particle Reynolds number of these particles, and 9 consequently the Nusselt number.

10 In contrast, application of the simplified mapping method makes the deviation even larger (see 11 panel $b$ in Figure $\mathrm{C} 1$ ). This is due to that fact that the local particle volume fraction fluctuates 12 strongly, and consequently the local Reynolds and heat transfer coefficient cannot be captured 13 accurately.

\section{Figure C1}

15 In order to be assured that the temperature is accurately calculated using the Laplace 16 transformation, a scenario with zero heat source was considered. The result of this scenario was 17 then compared with the temperature profile from the Schumann solution. As shown in Figure $18 \mathrm{C} 2$, the temperatures obtained using both methodologies are identical. 
Table C.1-Simulation conditions for the CFD-DEM simulations and benchmark.

\begin{tabular}{|c|c|c|c|c|c|}
\hline \multicolumn{3}{|c|}{ Catalytic Bed Dimensions } & \multicolumn{3}{|c|}{ Solid Phase Properties } \\
\hline Height & $m$ & 0.6 & $d_{p}$ & $m$ & 0.022 \\
\hline Length & $m$ & 0.1 & $\lambda_{p}$ & $W / K m$ & 0.1 \\
\hline \multirow[t]{2}{*}{ Width } & $m$ & 0.1 & $C_{p, p}$ & $\mathrm{~J} / \mathrm{kgK}$ & 5 \\
\hline & & & $\rho_{p}$ & $\mathrm{~kg} / \mathrm{m}^{3}$ & 1000 \\
\hline \multicolumn{3}{|c|}{ Gas Phase Properties } & \multicolumn{3}{|c|}{ Initial Condition } \\
\hline$C_{p, f}$ & $J^{J} / g_{K}$ & 1007 & $T_{g 0}$ & $K$ & 300 \\
\hline$\rho_{f}$ & $\mathrm{~kg} / \mathrm{m}^{3}$ & 1.188 & $T_{p 0}$ & $K$ & 300 \\
\hline$v_{f}$ & $\mathrm{~m}^{2} / \mathrm{s}$ & $1.5 \cdot 10^{-5}$ & \multicolumn{3}{|c|}{ Boundary Condition } \\
\hline$\lambda_{f}$ & $W / K m$ & 0.0256 & $T_{g i}$ & $K$ & 330 \\
\hline $\begin{array}{l}\text { Prandtl } \\
\text { number }\end{array}$ & - & 0.70097 & $U$ & $\mathrm{~m} / \mathrm{s}$ & 0.1 \\
\hline \multicolumn{3}{|c|}{ Contact Model Parameters for DEM } & \multicolumn{3}{|c|}{ Numerical Simulation Parameters } \\
\hline $\bar{Y}$ & $N / m^{2}$ & $2 \cdot 10^{5}$ & $\Delta t_{C F D}$ & s & 0.025 \\
\hline$v$ & - & 0.45 & $\Delta t_{D E M}$ & s & $1.25 \cdot 10^{-4}$ \\
\hline$\mu_{c, p}$ & - & 1 & $t_{\text {sim }}$ & s & 20 \\
\hline$e_{p p}$ & - & 1 & $\Delta x_{c e l l}$ & $m$ & 0.044 \\
\hline$\mu_{c, w}$ & - & 0.5 & \multirow{2}{*}{\multicolumn{2}{|c|}{$\begin{array}{l}\text { time discretization scheme } \\
\text { spatial discretization scheme }\end{array}$}} & implicit-explicit \\
\hline$e_{w p}$ & - & 0.3 & & & $2^{\text {nd }}$ order \\
\hline
\end{tabular}

\section{References}

4 Kloss, C., Goniva, C., Hager, A., Amberger, S., Pirker, S., 2012. Models, algorithms and 5 validation for opensource DEM and CFD-DEM. Progress in Computational Fluid Dynamics, 6 an International Journal 12, 140-152. 


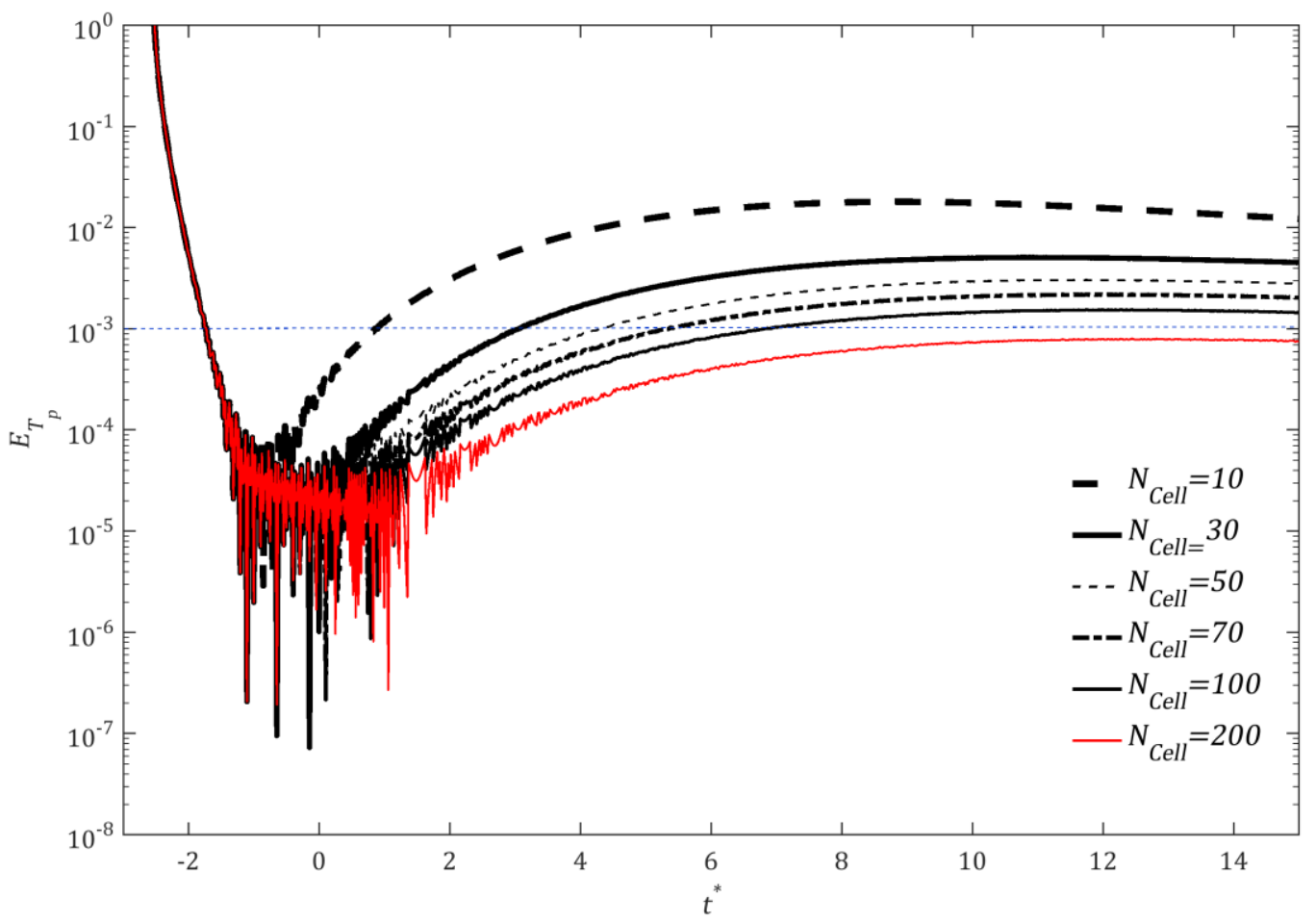

3 Figure 1. Error associated with the numerical solution for the particle temperature for a variety of grid resolutions and as a function of the dimensionless time. 

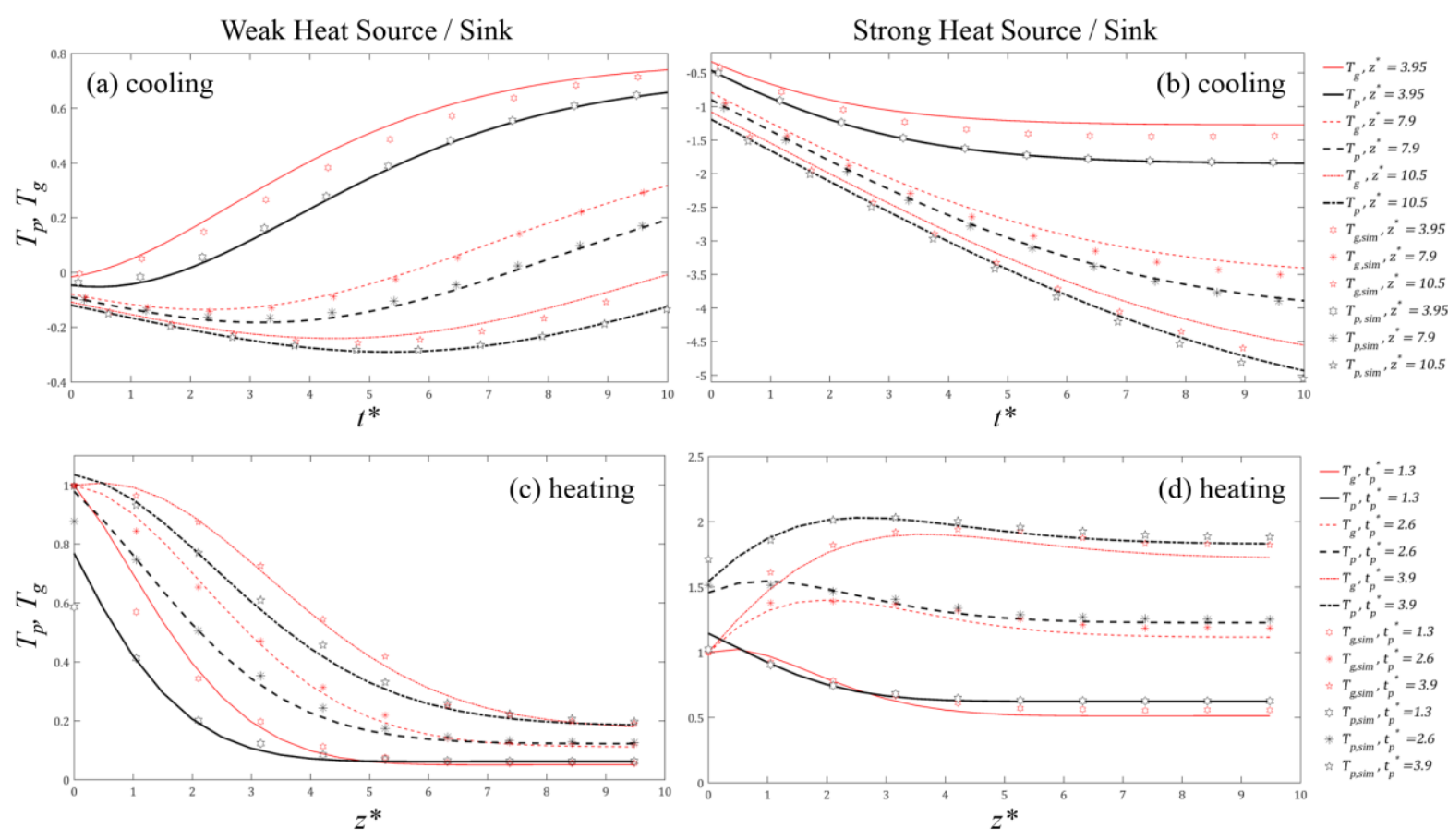

2 Figure 2. Comparison of the predicted gas and particle temperatures using CFD-DEM with

3 the corresponding analytical values as a function of time and the cooling rate (panel a:

4 weak cooling with $h_{q}^{*}=-0.05$; panel b: strong cooling with $\left.h_{q}^{*}=-0.5\right)$, as well as the

$5 \quad$ heating rate as a function of the bed position (panel c: weak heating with $h_{q}^{*}=0.05$, panel

$6 \quad d$ : strong heating with $h_{q}^{*}=0.5$ ). The lines correspond to the analytical solution, whereas 

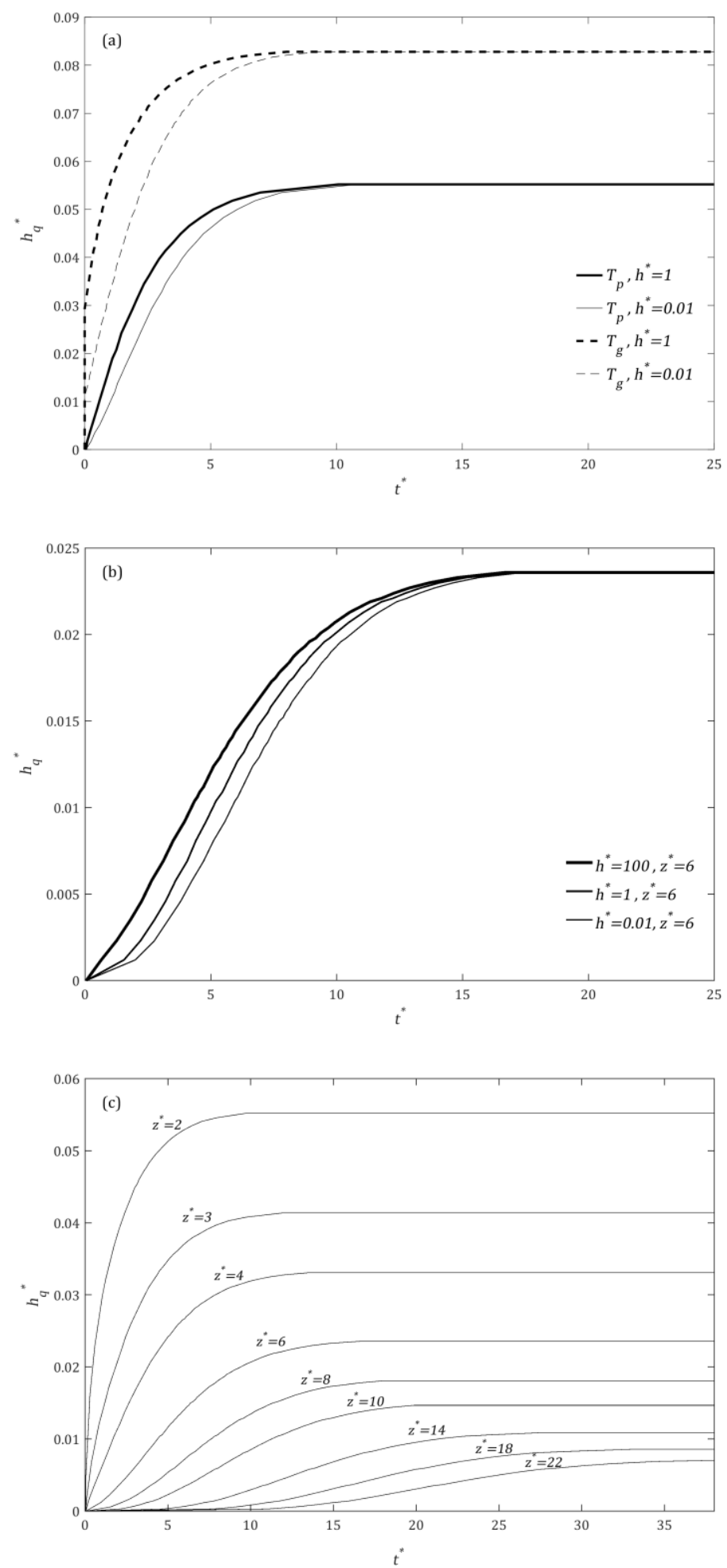

2 Figure 3. Map to quantify the validity of Schumann's solution. Panel a: comparison of the 3 map for gas and particle temperatures at $z^{*}=2$. Panel $b$ : the effect of the heat capacity 4 ratio on the particle temperature map for $z^{*}=6$. Panel c: the effect of the dimensionless 

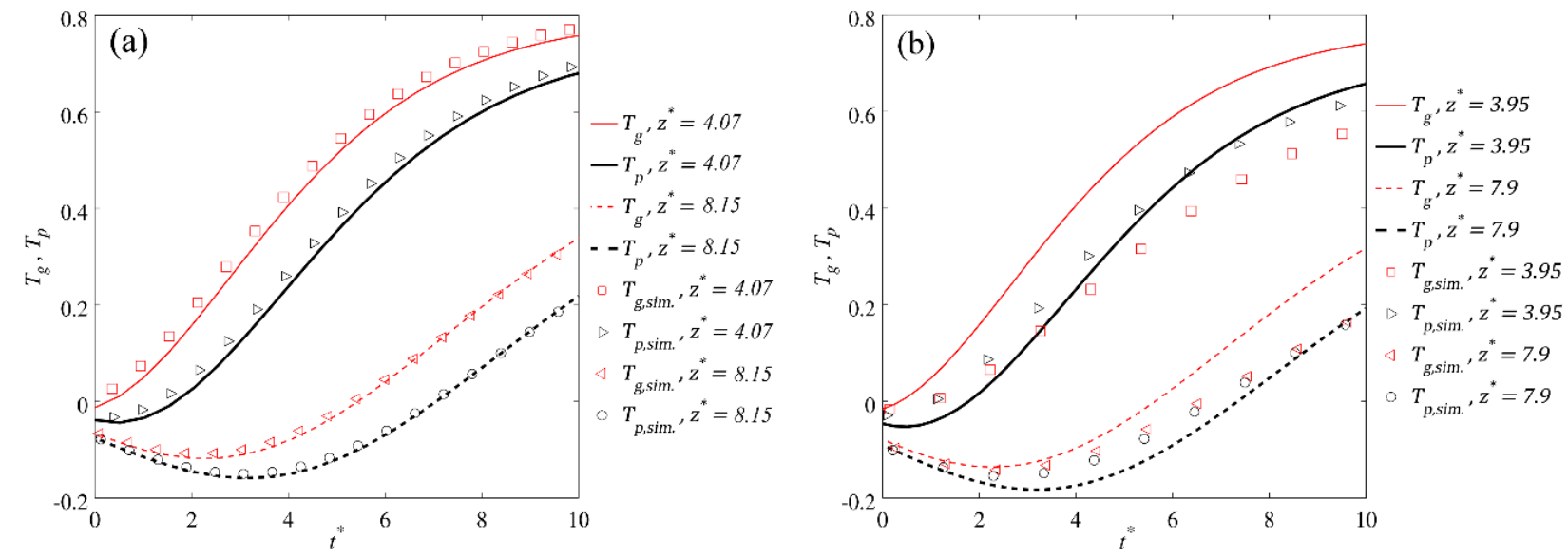

$3 \quad$ Figure C1 - Comparison of the predicted temperatures (using CFDEM®) with the analytical

4 solution for $h_{q}=0.05$ using a) a perfect lattice arrangement of the particles, as well as b) a simplified mapping method for local voidage calculation.

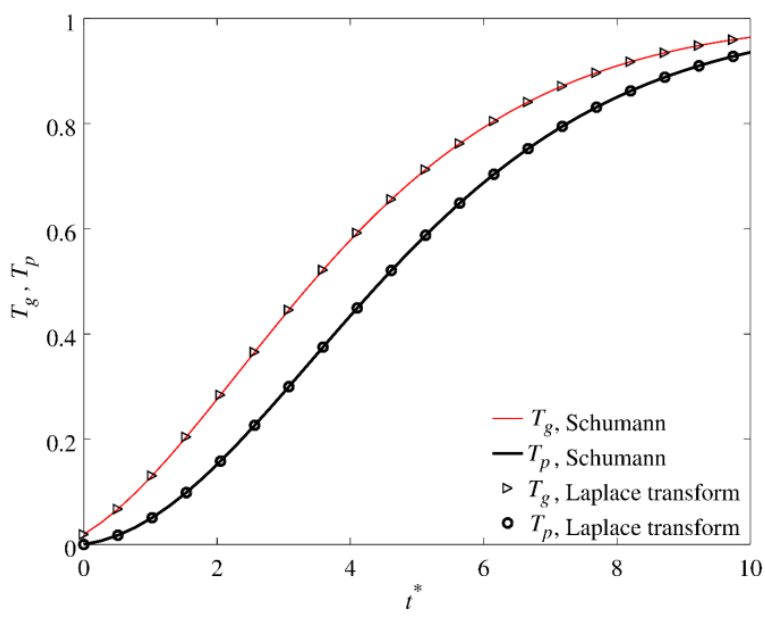

